Pacific

Journal of

Mathematics

REMOVABLE SETS FOR SUBHARMONIC FUNCTIONS

Juhani Rinhentaus

Volume $194 \quad$ No. 1

May 2000 


\title{
REMOVABLE SETS FOR SUBHARMONIC FUNCTIONS
}

\author{
JUHANI RIIHENTAUS
}

\begin{abstract}
It is a classical result that a closed exceptional polar set is removable for subharmonic functions which are bounded above. Gardiner has shown that in the case of a compact exceptional set the above boundedness condition can be relaxed by imposing certain smoothness and Hausdorff measure conditions on the set. We give related results for a closed exceptional set, by replacing the smoothness and Hausdorff measure conditions with one sole condition on Minkowski upper content.
\end{abstract}

\section{Introduction.}

In the sequel $\Omega$ is always an open set in $\mathbb{R}^{n}, n \geq 2$, and $E \subset \Omega$ is closed in $\Omega$. It is a classical result [HK, Theorem 5.18, p. 237] that if $f$ is subharmonic in $\Omega \backslash E$ and bounded above and moreover $E$ is polar, then $f$ has a subharmonic extension to the whole of $\Omega$. Imposing certain constraints on the geometry and size of the set $E$, Gardiner relaxed considerably the boundedness requirement of $f$ [Ga, Theorems 1 and 3, pp. 71-74]. To state his results, let $\Phi: \Omega \rightarrow \mathbb{R}$ be a $\mathcal{C}^{2}$ function with nonvanishing gradient throughout $\Omega$. Put $S=\{x \in \Omega: \Phi(x)=0\}$. Write $d(x, S)$ for the distance from $x \in \mathbb{R}^{n}$ to $S$ and let $\Lambda_{\alpha}$ be the $\alpha$-dimensional Hausdorff (outer) measure in $\mathbb{R}^{n}$.

Theorem A. Let $\alpha \in(0, n-2)$ and $E$ be a compact subset of $S$ such that $\Lambda_{\alpha}(E)=0$. If $f$ is subharmonic in $\Omega \backslash E$ and satisfies

$$
f(x) \leq C d(x, S)^{\alpha+2-n} \quad(x \in \Omega \backslash S)
$$

for some positive constant $C$, then $f$ has a subharmonic extension to $\Omega$.

Theorem B. Let $\alpha \in(0, n-2)$ and $E$ be a compact subset of $S$ such that $\Lambda_{\alpha}(E)<\infty$. If $f$ is subharmonic in $\Omega \backslash E$ and satisfies

$$
f(x) \leq u(d(x, S)) \quad(x \in \Omega \backslash S)
$$

where $t^{n-2-\alpha} u(t) \rightarrow 0(t \rightarrow 0+)$, then $f$ has a subharmonic extension to $\Omega$.

Our notation is more or less standard or will be explained below. For example, $B(x, r)$ is the open ball in $\mathbb{R}^{n}$, with center $x$ and radius $r$. The family of test functions on $\Omega$ is denoted by $\mathcal{D}(\Omega)$. The differential operator $\left(\mathcal{D}_{1}\right)^{\lambda_{1}} \cdots\left(\mathcal{D}_{n}\right)^{\lambda_{n}}=\left(\frac{\partial}{\partial x_{1}}\right)^{\lambda_{1}} \cdots\left(\frac{\partial}{\partial x_{n}}\right)^{\lambda_{n}}$ is denoted by $\mathcal{D}^{\lambda}$. Here $\lambda=$ 
$\left(\lambda_{1}, \ldots, \lambda_{n}\right) \in(\mathbb{N} \cup\{0\})^{n}$ is a multi-index, and $|\lambda|=\lambda_{1}+\cdots+\lambda_{n}$. The Laplacian is $\Delta=\mathcal{D}_{1}^{2}+\cdots+\mathcal{D}_{n}^{2}$. The notation $C(n, \alpha, \ldots)$, say, means that $C$ is a constant depending only on $n, \alpha, \ldots$. As usual, constants may vary from line to line.

Gardiner also shows [Ga, Theorems 2 and 4, pp. 72-73] that his results are sharp in the following sense: If one drops the smoothness assumption $E \subset S$ then the exceptional set $E$ is not any more necessarily removable. Our purpose is to point out that there exist, however, results which are in a certain sense parallel to Gardiner's results but where no smoothness conditions are necessary to impose on the exceptional set. As a matter of fact, we show below in Theorems 1 and 2 that results similar to Gardiner's hold when his conditions,

(i) $E \subset S$ where $S$ is a $\mathcal{C}^{2}(n-1)$-dimensional manifold in $\Omega$,

(ii) $\Lambda_{\alpha}(E)=0\left(\operatorname{resp} . \Lambda_{\alpha}(E)<\infty\right)$,

are replaced by one geometric measure condition $M^{\alpha}(E)=0\left(\right.$ resp. $M^{\alpha}(E)$ $<\infty)$ where $M^{\alpha}$ is the upper Minkowski content. Our proofs are different and perhaps shorter than those of Gardiner. Moreover, our approach does not require the exceptional set $E$ to be compact, unlike in Gardiner's results. On the other hand, as is shown in Examples 1 and 2 below, Gardiner's and our results are independent: Neither our nor Gardiner's results are included in the other's.

Gardiner also [Ga, Theorem 5, p. 74] proves the following result:

Theorem C. Let $\alpha \in(0, n-2)$ and $E$ be a compact subset of $S$ such that $\Lambda_{\alpha}(E)=0$. If $f$ is subharmonic in $\Omega \backslash E$ and satisfies

$$
\mathcal{A}\left(f^{+}, x, r\right) \leq C r^{\alpha+2-n} \quad(\overline{B(x, r)} \subset \Omega)
$$

then $f$ has a subharmonic extension to $\Omega$.

Here $f^{+}=\max \{f, 0\}$ and $\mathcal{A}\left(f^{+}, x, r\right)$ is the mean value of $f^{+}$over the ball $B(x, r)$, with respect to the Lebesgue measure $m$ in $\mathbb{R}^{n}$.

Below in Theorem 3 we improve this result by dropping the condition that $E$ is compact. Again our approach is essentially different than that of Gardiner.

\section{Net measure and Minkowski content.}

For readers' convenience we first recall certain basic facts concerning net measure and Minkowski content and their relationship with the standard Hausdorff measure. For a more thorough discussion see e.g., [HP, pp. 4144] and [Fa, pp. 33, 42]. 
Let $A \subset \mathbb{R}^{n}$ and $\alpha \in[0, n]$. For each $\epsilon>0$ define

$$
\mathcal{L}_{\alpha}^{\epsilon}(A)=\inf \sum_{i=1}^{\infty} s_{i}^{\alpha}
$$

where the infimum is over all coverings of $A$ by countable disjoint collection of dyadic cubes $Q_{i}$ with (side)length $s_{i} \leq \epsilon$. Define the $\alpha$-dimensional net measure of $A$ by

$$
\mathcal{L}_{\alpha}(A)=\lim _{\epsilon \rightarrow 0+} \mathcal{L}_{\alpha}^{\epsilon}(A) .
$$

It is well-known that the standard Hausdorff measure $\Lambda_{\alpha}$ and the net measure $\mathcal{L}_{\alpha}$ are comparable: There are positive constants $C_{1}=C_{1}(n)$ and $C_{2}=C_{2}(n)$ such that

$$
C_{1} \mathcal{L}_{\alpha}(A) \leq \Lambda_{\alpha}(A) \leq C_{2} \mathcal{L}_{\alpha}(A)
$$

for all $A \subset \mathbb{R}^{n}$.

To define the Minkowski content, let $A \subset \mathbb{R}^{n}, \alpha \in[0, n]$ and $\epsilon>0$. Write

$$
A_{\epsilon}=\left\{x \in \mathbb{R}^{n}: d(x, A)<\epsilon\right\} .
$$

The $\alpha$-dimensional upper Minkowski content of $A$ is defined by

$$
\mathcal{M}^{\alpha}(A)=\limsup _{\epsilon \rightarrow 0+} \frac{m\left(A_{\epsilon}\right)}{\epsilon^{n-\alpha}} .
$$

It is well-known that there is a positive constant $C_{3}=C_{3}(n, \alpha)$ such that

$$
C_{3} \Lambda_{\alpha}(A) \leq \mathcal{M}^{\alpha}(A)
$$

for all $A \subset \mathbb{R}^{n}$. The reverse inequality does not hold in general, but is true for certain smooth sets, even for $\alpha$ rectifiable closed subsets of $\mathbb{R}^{n}$ (here $\alpha$ is a positive integer). See [HP, p. 41] and [Fe, 3.2.39, p. 275].

Our argument will essentially be based on the following type of partition of unity, see [HP, Lemma 3.1, p. 43]:

Lemma. Let $\left\{Q_{i}: i=1, \ldots, N\right\}$ be a finite disjoint collection of dyadic cubes of length $s\left(Q_{i}\right)=s_{i}$. For each $i$, there is a function $\varphi_{i} \in \mathcal{C}_{0}^{\infty}\left(\mathbb{R}^{n}\right)$ with support spt $\varphi_{i} \subset \frac{3}{2} Q_{i}$ such that $\sum_{i=1}^{\infty} \varphi_{i}(x)=1$ for all $x \in \cup_{i=1}^{N} Q_{i}$. Furthermore, for each multi-index $\lambda$, there is a constant $C_{\lambda}=C_{\lambda}(\lambda, n)$ for which $\left|\mathcal{D}^{\lambda} \varphi_{i}(x)\right| \leq C_{\lambda} s_{i}^{-|\lambda|}$ for all $x \in \mathbb{R}^{n}$ and $i=1, \ldots, N$.

\section{The results.}

Our first result is parallel to Gardiner's Theorem A:

Theorem 1. Suppose that $\alpha \in[0, n-2]$ and $\mathcal{M}^{\alpha}(E)=0$. If $f$ is subharmonic in $\Omega \backslash E$ and satisfies

$$
f(x) \leq C^{*} d(x, E)^{\alpha+2-n} \quad(x \in \Omega \backslash E)
$$

for some positive constant $C^{*}$, then $f$ has a subharmonic extension to $\Omega$. 
Proof. If $\alpha=0$ then $E=\emptyset$. If $\alpha=n-2$, then $E$ is polar e.g., by [HK, Theorem 5.14, p. 288]. Since $f$ is then also bounded above, the claim follows from the classical result [HK, Theorem 5.18, p. 237].

It remains to consider the case $\alpha \in(0, n-2)$. Since $f^{+}$is subharmonic, and also

$$
f^{+}(x) \leq C^{*} d(x, E)^{\alpha+2-n} \quad(x \in \Omega \backslash E),
$$

we may suppose that $f \geq 0$.

We first show that $f \in \mathcal{L}_{\text {loc }}^{1}(\Omega)$, cf. [HP, p. 42] and [Ri, pp. 730-731]. It is sufficient to show that for some $r>0$,

$$
\int_{E_{r}} f d m<\infty
$$

Take $\epsilon>0$ arbitrarily. Since $\mathcal{M}^{\alpha}(E)=0$, there is $r_{o}, 0<r_{o}<1$, such that $m\left(E_{r}\right) \leq \epsilon r^{n-\alpha}$ for all $r, 0 \leq r \leq r_{o}$. Take any such $r$, and write for each $j=0,1, \ldots$,

$$
K_{j}=\left\{x \in \mathbb{R}^{n}: d(x, E)<r 2^{-j}\right\} .
$$

Then

$$
E_{r}=\bigcup_{j=0}^{\infty}\left(K_{j} \backslash K_{j+1}\right)
$$

and

$$
\begin{aligned}
\int_{E_{r}} f(x) d m(x) & \leq C^{*} \int_{E_{r}} d(x, E)^{\alpha+2-n} d m(x) \\
& =C^{*} \sum_{j=0}^{\infty} \int_{K_{j} \backslash K_{j+1}} d(x, E)^{\alpha+2-n} d m(x) \\
& \leq C^{*} \sum_{j=0}^{\infty}\left[r 2^{-(j+1)}\right]^{\alpha+2-n} m\left(K_{j}\right) \\
& \leq C^{*} 2^{n-2-\alpha} r^{\alpha+2-n} \sum_{j=0}^{\infty} 2^{(n-2-\alpha) j} \epsilon\left(r 2^{-j}\right)^{n-\alpha} \\
& \leq C^{*} 2^{n-2-\alpha} r^{2} \epsilon \sum_{j=0}^{\infty} 2^{-2 j}<\infty
\end{aligned}
$$

Thus $f \in \mathcal{L}_{\text {loc }}^{1}(\Omega)$. For later use we observe that we also got

$$
\int_{E_{r}} d(x, E)^{\alpha+2-n} d m(x) \leq C r^{2} \epsilon
$$

for all $r, 0 \leq r \leq r_{o}$, where $C=C\left(n, \alpha, C^{*}\right)$. 
To complete the proof, it remains to show that for any nonnegative test function $\varphi \in \mathcal{D}(\Omega)$,

$$
\int f \Delta \varphi d m \geq 0 \text {. }
$$

We may suppose that $0 \leq \varphi \leq 1$ and $\left|\mathcal{D}^{\lambda} \varphi\right| \leq 1$ for each multi-index $\lambda$, $|\lambda| \leq 2$. Compare [KW, p. 113].

Let $K=\operatorname{spt} \varphi$. We may suppose that $K_{r_{o}} \subset \Omega$. Choose $s=2^{-k}$ so small that $3 s \sqrt{n} \leq r_{o}$. Cover $K$ by a finite, disjoint collection of dyadic cubes $Q_{i}$ with length $s\left(Q_{i}\right)=s, i=1, \ldots, N$. We may suppose that

$$
\frac{3}{2} Q_{i} \cap E \neq \emptyset \text { for } i=1, \ldots, N^{*},
$$

and

$$
\frac{3}{2} Q_{i} \cap E=\emptyset \text { for } i=N^{*}+1, \ldots, N,
$$

for some $N^{*} \in \mathbb{N}, 1 \leq N^{*} \leq N$. Let $\varphi_{i}, i=1, \ldots, N$, be the test functions related to the collection $Q_{i}, i=1, \ldots, N$, and possessing the properties described in the above Lemma.

Since $f$ is subharmonic in $\Omega \backslash E$ and all $\varphi \varphi_{i}, i=N^{*}+1, \ldots, N$, are nonnegative test functions in $\mathcal{D}(\Omega \backslash E)$, we have

$$
\int f \Delta\left(\varphi \varphi_{i}\right) d m \geq 0 \text { for } i=N^{*}+1, \ldots, N .
$$

In view of these inequalities, we get

$$
\begin{aligned}
\int f \Delta \varphi d m & =\int f \Delta\left[\varphi\left(\sum_{j=1}^{N} \varphi_{i}\right)\right] d m=\sum_{i=1}^{N} \int_{\frac{3}{2} Q_{i}} f \Delta\left(\varphi \varphi_{i}\right) d m \\
& \geq \sum_{i=1}^{N^{*}} \int_{\frac{3}{2} Q_{i}} f \Delta\left(\varphi \varphi_{i}\right) d m .
\end{aligned}
$$

An easy computation shows that

$$
\Delta\left(\varphi \varphi_{i}\right)=(\Delta \varphi) \varphi_{i}+\varphi\left(\Delta \varphi_{i}\right)+2 \sum_{j=1}^{n} \mathcal{D}_{j} \varphi \mathcal{D}_{j} \varphi_{i}
$$

By the properties of the test functions $\varphi_{i}$ and $\varphi$, we have for all $i=1, \ldots, N^{*}$ and $x \in \mathbb{R}^{n}$,

$$
\begin{aligned}
\left|\Delta\left(\varphi \varphi_{i}\right)(x)\right| & \leq|\Delta \varphi(x)|\left|\varphi_{i}(x)\right|+|\varphi(x)|\left|\Delta \varphi_{i}(x)\right|+2 \sum_{j=1}^{n}\left|\mathcal{D}_{j} \varphi(x)\right|\left|\mathcal{D}_{j} \varphi_{i}(x)\right| \\
& \leq 1+\frac{C_{2}}{s^{2}}+\frac{C_{1}}{s} \leq \frac{C}{s^{2}}
\end{aligned}
$$


where $C=C\left(n, C_{1}, C_{2}\right)$. The last inequality here follows from the fact that, since $0<r_{o}<1$, also $0<s<1$.

For each cube $Q_{i}, i=1, \ldots, N^{*}$, there are clearly at most $3^{n}$ cubes $Q_{j}$, $s\left(Q_{j}\right)=s, j=1, \ldots, N_{i} \leq 3^{n}$ (just the adjacent cubes to $Q_{i}$ with equal length), such that

$$
\frac{3}{2} Q_{i} \cap \frac{3}{2} Q_{j} \neq \emptyset
$$

Using this, the fact that $\frac{3}{2} Q_{i} \subset E_{3 s \sqrt{n}}, i=1, \ldots, N^{*},(3)$ and (4), we get

$$
\begin{aligned}
\int f \Delta \varphi d m & \geq-\frac{C}{s^{2}} \sum_{i=1}^{N^{*}} \int_{\frac{3}{2} Q_{i}} f d m=-\frac{C}{s^{2}} \sum_{i=1}^{N^{*}} \int_{E_{3 s \sqrt{n}}} f \chi_{\frac{3}{2} Q_{i}} d m \\
& =-\frac{C}{s^{2}} \int_{E_{3 s \sqrt{n}}} f\left(\sum_{i=1}^{N^{*}} \chi_{\frac{3}{2} Q_{i}}\right) d m \\
& \geq-\frac{3^{n} C}{s^{2}} \int_{E_{3 s \sqrt{n}}} f d m .
\end{aligned}
$$

Here $\chi_{\frac{3}{2} Q_{i}}$ is the characteristic function of $\frac{3}{2} Q_{i}, i=1, \ldots, N^{*}$. Above we have used the fact that $\sum_{i=1}^{N^{*}} \chi_{\frac{3}{2} Q_{i}}(x) \leq 3^{n}$ for all $x \in E_{3 s \sqrt{n}}$. Indeed, if $x \notin \frac{3}{2} Q_{i}$ for $i=1, \ldots, N^{*}$, then $\sum_{i=1}^{N^{*}} \chi_{\frac{3}{2}} Q_{i}(x)=0$. If $x \in \frac{3}{2} Q_{i_{o}}$ for some $i_{o}$, $1 \leq i_{o} \leq N^{*}$, then by (5) we see that among the cubes $\frac{3}{2} Q_{i}, i=1, \ldots, N^{*}$, there are at most $N_{i_{o}}$ such for which $x \in \frac{3}{2} Q_{i}$. Since $N_{i_{o}} \leq 3^{n}$ (see (5) above), also $\sum_{i=1}^{N^{*}} \chi_{\frac{3}{2} Q_{i}}(x) \leq 3^{n}$. Proceeding further then, and using also (2), we get

$$
\begin{aligned}
\int f \Delta \varphi d m & \geq-\frac{C}{s^{2}} \int_{E_{3 s \sqrt{n}}} f d m \\
& \geq-\frac{C}{s^{2}} \int_{E_{3 s \sqrt{n}}} d(x, E)^{\alpha+2-n} d m(x) \\
& \geq-\frac{C}{s^{2}}(3 s \sqrt{n})^{2} \epsilon=-C \epsilon .
\end{aligned}
$$

Since $\epsilon>0$ was arbitrary and $C=C\left(n, \alpha, C^{*}\right)$, it follows that

$$
\int f \Delta \varphi d m \geq 0
$$

concluding the proof.

As Gardiner points out [Ga, p. 73], a slight modification of his proof of Theorem A yields Theorem B. In our frame the situation is similar: 
Theorem 2. Suppose that $\alpha \in[0, n-2]$ and $\mathcal{M}^{\alpha}(E)<\infty$. If $f$ is subharmonic in $\Omega \backslash E$ and satisfies

$$
f(x) \leq u(d(x, E)) \quad(x \in \Omega \backslash E)
$$

where $u(t)$ is a Borel measurable function such that $t^{n-2-\alpha} u(t) \rightarrow 0(t \rightarrow$ $0+)$, then $f$ has a subharmonic extension to $\Omega$.

The proof goes along the same lines as above with only minor changes. In fact, take $\epsilon>0$ arbitrarily. Choose then $r_{o}, 0<r_{o}<1$, such that

$$
u(t)<\epsilon t^{\alpha+2-n}
$$

whenever $0<t<r_{o}$. Since $\mathcal{M}^{\alpha}(E)<\infty$, we may suppose that $m\left(E_{r}\right)<$ $M r^{n-\alpha}$ for all $r, 0<r \leq r_{o}$. Proceeding then as in the proof of Theorem 1 (see (2) above), one sees that for all $r, 0<r \leq r_{o}$,

$$
\begin{aligned}
\int_{E_{r}} f(x) d m(x) & \leq \int_{E_{r}} u(d(x, E)) d m(x) \leq \epsilon \int_{E_{r}} d(x, E)^{\alpha+2-n} d m(x) \\
& <\epsilon C r^{2} M<\infty .
\end{aligned}
$$

The rest of the proof goes as in the proof of Theorem 1 .

Example 1. Let $0<\alpha<1$ be arbitrarily given. By [Fa, Example 4.5, p. 58] there is a uniform Cantor set $F \subset[0,1]$ such that $\mathcal{M}^{\alpha}(F)=0$. Set $E=F \times \cdots \times F$. Then $E$ is closed and by [Fa, Example 7.6, p. 95], $\mathcal{M}^{\alpha n}(E)=0$. Clearly $E$ is not contained in any $\mathcal{C}^{2}(n-1)$-dimensional manifold. Thus our results, Theorems 1 and 2 above, can be applied in situations where Gardiner's Theorems A and B cannot be used.

Example 2. By $[\mathbf{K o}, 2.3$, p. 462] there is for each $\alpha, 0<\alpha<2$, a countable, compact subset $F$ of the complex plane $\mathbb{C}$ with $\mathcal{M}^{\alpha}(F)>0$. Let $E=F \times\{0\} \subset \mathbb{R}^{3}$. One sees easily that $\mathcal{M}^{\alpha}(E)>0$. Since $E$ is countable, $\Lambda_{\alpha}(E)=0$. Thus we have an example where Gardiner's theorems can be used whereas our results are not applicable.

Our last theorem improves Gardiner's Theorem C by allowing the exceptional set to be noncompact. The proof we present is different from that of Gardiner.

Theorem 3. Suppose that $\alpha \in[0, n-2]$ and $\Lambda_{\alpha}(E)=0$. If $f$ is subharmonic in $\Omega \backslash E$ and satisfies

$$
\mathcal{A}\left(f^{+}, x, r\right) \leq C^{*} r^{\alpha+2-n} \quad(\overline{B(x, r)} \subset \Omega)
$$

for some positive constant $C^{*}$, then $f$ has a subharmonic extension to $\Omega$.

Proof. As in the proof of Theorem 1, we may suppose that $\alpha \in(0, n-2)$ and $f \geq 0$. Since $f \in \mathcal{L}_{l o c}^{1}(\Omega)$, it is sufficient to show that

$$
\int f \Delta \varphi d m \geq 0
$$


for any nonnegative test function $\varphi \in \mathcal{D}(\Omega)$. Take such a $\varphi$ arbitrarily. As in the proof of Theorem 1, we may suppose that $0 \leq \varphi \leq 1$ and $\left|\mathcal{D}^{\lambda} \varphi\right| \leq 1$ for each multi-index $\lambda,|\lambda| \leq 2$. Let $K=\operatorname{spt} \varphi$. Choose $r_{o}, 0<r_{o}<1$, such that $\widehat{K}=\bar{K}_{r_{o}} \subset K_{2 r_{o}} \subset \bar{K}_{2 r_{o}} \subset \Omega$. Let $\epsilon>0$ be arbitrarily given. We will cover $K$ by a suitable collection of mutually disjoint dyadic cubes. This will be done in three steps.

First, using the assumption $\Lambda_{\alpha}(E)=0$ and (1), we find a sequence of mutually disjoint dyadic cubes $Q_{i}, s\left(Q_{i}\right)=s_{i}, i=1,2, \ldots$, such that

$$
\sum_{i=1}^{\infty} s_{i}^{\alpha}<\epsilon
$$

We may suppose that $3 s_{i} \sqrt{n}<r_{o}, i=1,2, \ldots$ Since $E \cap \widehat{K}$ is compact, there is $N_{1} \in \mathbb{N}$ such that

$$
E \cap \widehat{K} \subset \bigcup_{i=1}^{N_{1}} Q_{i}
$$

Second, we attach to each cube $Q_{i}, s\left(Q_{i}\right)=s_{i}, i=1, \ldots, N_{1}$, all adjacent dyadic cubes with the same length $s_{i}$. Since two dyadic cubes are either mutually disjoint or one is contained in the other, we may drop extra cubes away. Proceeding in this way we get a collection of mutually disjoint cubes $Q_{i}^{j_{i}}, j_{i}=0, \ldots, n_{i}, i=1, \ldots, N_{1}$, such that

$$
s\left(Q_{i}^{j_{i}}\right)=s\left(Q_{i}\right)=s_{i}, \quad j_{i}=0, \ldots, n_{i} \leq 3^{n}-1, i=1, \ldots, N_{1} .
$$

(That indeed $n_{i} \leq 3^{n}-1$ for all $i=1, \ldots, N_{1}$, follows just from the fact that we are considering adjacent cubes of the same length.)

Third, cover the remaining bounded set $K \backslash\left(\left(\cup_{i=1}^{N_{1}} Q_{i}\right) \cup\left(\cup_{i=1}^{N_{1}}\left(\cup_{j_{i}=0}^{n_{i}} Q_{i}^{j_{i}}\right)\right)\right)$ by mutually disjoint, dyadic cubes $\widetilde{Q}_{k}$, all with the same length $s\left(\widetilde{Q}_{k}\right)=s$, $k=0, \ldots, N_{2}$, where $s=\min \left\{s_{i}: i=1, \ldots, N_{1}\right\}$. Using then the facts that $Q_{i}$ and $Q_{i}^{j_{i}}$ are adjacent, that $s\left(Q_{i}\right)=s\left(Q_{i}^{j_{i}}\right)=s_{i}, j_{i}=0, \ldots, n_{i}$, and $s\left(\widetilde{Q}_{k}\right)=s \leq s_{i}, i=1, \ldots, N_{1}, k=0, \ldots, N_{2}$, one sees easily that

$$
\frac{3}{2} \widetilde{Q}_{k} \cap E=\emptyset \text { for } k=0, \ldots, N_{2} \text {. }
$$

In order to show that (6) holds, we next choose nonnegative test functions $\varphi_{i}, \varphi_{i}^{j_{i}}, j_{i}=0, \ldots, n_{i}, i=1, \ldots, N_{1}$, and $\widetilde{\varphi}_{k}, k=0, \ldots, N_{2}$, from $\mathcal{D}(\Omega)$ with the aid of the above Lemma, and thus with the following properties:

$$
\operatorname{spt} \varphi_{i} \subset \frac{3}{2} Q_{i}, \quad\left|\mathcal{D}^{\lambda} \varphi_{i}\right| \leq \frac{C_{\lambda}}{s_{i}^{|\lambda|}} \text { for } \lambda,|\lambda| \leq 2, i=1, \ldots, N_{1} ;
$$


(12)

$$
\begin{aligned}
\operatorname{spt} \varphi_{i}^{j_{i}} \subset \frac{3}{2} Q_{i}^{j_{i}},\left|\mathcal{D}^{\lambda} \varphi_{i}^{j_{i}}\right| & \leq \frac{C_{\lambda}}{s_{i}^{|\lambda|}} \\
& \text { for } \lambda,|\lambda| \leq 2, j_{i}=0, \ldots, n_{i} ; i=1, \ldots, N_{1}
\end{aligned}
$$

$$
\operatorname{spt} \widetilde{\varphi}_{k} \subset \frac{3}{2} \widetilde{Q}_{k}, \quad\left|\mathcal{D}^{\lambda} \widetilde{\varphi}_{k}\right| \leq \frac{C_{\lambda}}{s^{|\lambda|}} \text { for } \lambda,|\lambda| \leq 2, k=0, \ldots, N_{2}
$$

$$
\sum_{i=1}^{N_{1}} \varphi_{i}(x)+\sum_{i=1}^{N_{1}} \sum_{j_{i}=0}^{n_{i}} \varphi_{i}^{j_{i}}(x)+\sum_{k=0}^{N_{2}} \widetilde{\varphi}_{k}(x)=1 \text { for } x \in K .
$$

Using then (10), (13) and the fact that $f$ is subharmonic in $\Omega \backslash E$, one gets

$$
\int_{\frac{3}{2} \widetilde{Q}_{k}} f \Delta\left(\varphi \widetilde{\varphi}_{k}\right) d m \geq 0 \text { for } k=0, \ldots, N_{2} .
$$

From this, (14), (11) and (12), it follows that

$$
\begin{aligned}
\int f \Delta \varphi d m & =\int f \Delta\left[\varphi\left(\sum_{i=1}^{N_{1}} \varphi_{i}+\sum_{i=1}^{N_{1}} \sum_{j_{i}=0}^{n_{i}} \varphi_{i}^{j_{i}}+\sum_{k=0}^{N_{2}} \widetilde{\varphi}_{k}\right)\right] d m \\
& \geq \sum_{i=1}^{N_{1}} \int_{\frac{3}{2} Q_{i}} f \Delta\left(\varphi \varphi_{i}\right) d m+\sum_{i=1}^{N_{1}} \sum_{j_{i}=0}^{n_{i}} \int_{\frac{3}{2} Q_{i}^{j_{i}}} f \Delta\left(\varphi \varphi_{i}^{j_{i}}\right) d m .
\end{aligned}
$$

Using then (11) and (12) and proceeding as in the proof of Theorem 1, we get similar estimates as in (4),

$$
\left|\Delta\left(\varphi \varphi_{i}\right)(x)\right| \leq \frac{C}{s_{i}^{2}} \text { for } x \in \frac{3}{2} Q_{i}, i=1, \ldots, N_{1}
$$

and

$$
\left|\Delta\left(\varphi \varphi_{i}^{j_{i}}\right)(x)\right| \leq \frac{C}{s_{i}^{2}} \text { for } x \in \frac{3}{2} Q_{i}^{j_{i}}, j_{i}=0, \ldots, n_{i}, i=1, \ldots, N_{1}
$$

In view of these inequalities, and of (8), (9) and (7), we get (in the sequel $x_{i}$ and $x_{i}^{j_{i}}$ are the centers of the cubes $Q_{i}, Q_{i}^{j_{i}}, j_{i}=0, \ldots, n_{i}, i=1, \ldots, N_{1}$, 
respectively, and $\left.\nu_{n}=m(B(0,1))\right)$

$$
\begin{aligned}
& \int f \Delta \varphi d m \\
& \geq-C\left(\sum_{i=1}^{N_{1}} \frac{1}{s_{i}^{2}} \int_{\frac{3}{2} Q_{i}} f d m+\sum_{i=1}^{N_{1}} \sum_{j_{i}=0}^{n_{i}} \frac{1}{s_{i}^{2}} \int_{\frac{3}{2} Q_{i}^{j_{i}}} f d m\right) \\
& \geq-C\left(\sum_{i=1}^{N_{1}} \frac{1}{s_{i}^{2}} \int_{B\left(x_{i}, \frac{3}{4} s_{i} \sqrt{n}\right)} f d m+\sum_{i=1}^{N_{1}} \sum_{j_{i}=0}^{n_{i}} \frac{1}{s_{i}^{2}} \int_{B\left(x_{i}^{j_{i}}, \frac{3}{4} s_{i} \sqrt{n}\right)} f d m\right) \\
& \geq-\left(\frac{3}{4} \sqrt{n}\right)^{n} \nu_{n} C\left(\sum_{i=1}^{N_{1}} s_{i}^{\alpha}+3^{n} \sum_{i=1}^{N_{1}} s_{i}^{\alpha}\right)^{N_{1}}{ }^{\alpha} \geq-C \epsilon .
\end{aligned}
$$

Since $C=C\left(n, \alpha, C^{*}\right)$ and $\epsilon$ was arbitrarily given, (6) follows and the proof is complete.

\section{References}

[Fa] K.J. Falconer, Fractal Geometry, John Wiley \& Sons, 1993.

[Fe] H. Federer, Geometric Measure Theory, Springer-Verlag, 1969.

[Ga] S.J. Gardiner, Removable singularities for subharmonic functions, Pac. J. Math., 147 (1991), 71-80.

[HP] R. Harvey and J. Polking, Removable singularities of solutions of linear partial differential equations, Acta Math., 125 (1970), 39-56.

[HK] W.K. Hayman and P.B. Kennedy, Subharmonic Functions, I, Academic Press, 1976.

[KW] R. Kaufman and J.-M. Wu, Removable singularities for analytic or subharmonic functions, Ark. Mat., 18 (1980), 109-116.

[Ko] P. Koskela, Removable singularities for analytic functions, Michigan Math. J., 40 (1993), 459-466.

[Ri] J. Riihentaus, Removable singularities for Bloch and normal functions, Czech. Math. J., 43(118) (1993), 723-741.

Received September 9, 1998 and revised January 26, 1999.

South Carelia Polytechnic

FIN-53100 LAPPEENRANTA

FinLAND

E-mail address: juhani.riihentaus@mail.scp.fi 